\title{
"Combination-oriented molecular-targeting prevention" of cancer: a model involving the combination of TRAIL and a DR5 inducer
}

\author{
Tatsushi Yoshida • Mano Horinaka • \\ Toshiyuki Sakai
}

Received: 24 November 2009/Accepted: 11 December 2009/Published online: 6 January 2010

(C) The Japanese Society for Hygiene 2009

\begin{abstract}
Malignant tumors carry a high risk of death, and the prevention of malignant tumors is a crucial issue in preventive medicine. To this end, many chemopreventive agents have been tested, but the effects of single agents have been found to be insufficient to justify clinical trials. We have therefore hypothesized that combinations of different chemopreventive agents may synergistically enhance the preventive effect of chemopreventive agents used singly. To provide the treating physician with some guideline by which to choose the most effective agents to be combined, we propose a strategy which we have termed the "combination-oriented molecular-targeting prevention" of cancer. As the molecular target of our model, we focused on tumor necrosis factor-related apoptosis-inducing ligand (TRAIL), which specifically causes apoptosis in malignant tumor cells. Many of these agents were found to up-regulate the expression of death receptor 5, a TRAIL receptor. They were also found to synergistically induce apoptosis in malignant tumor cells when combined with TRAIL. Here, we strongly advocate that the strategy of "combination-oriented molecular-targeting prevention" of cancer will be a practical approach for chemoprevention against human malignant tumors.
\end{abstract}

Keywords Apoptosis - Cancer - Combination · DR5 - Prevention · TRAIL

T. Yoshida $\cdot$ M. Horinaka $\cdot$ T. Sakai $(\bowtie)$

Department of Molecular-Targeting Cancer Prevention, Graduate School of Medical Science, Kyoto Prefectural University of Medicine, Kawaramachi-Hirokoji,

Kamigyo-ku, Kyoto 602-8566, Japan

e-mail: tsakai@koto.kpu-m.ac.jp

\author{
Abbreviations \\ CHOP CCAAT/enhancer binding protein homologous \\ protein \\ DR5 Death receptor 5 \\ HDACI Histone deacetylase inhibitors \\ TRAIL Tumor necrosis factor-related apoptosis- \\ inducing ligand \\ TNF Tumor necrosis factor
}

\section{Introduction}

In Japan, malignant tumors are regarded as a very serious medical condition due to their association with a high mortality rate among patients. The prevention of malignant tumors is therefore an important focus of researchers in the field of preventive medicine. The administration of a single chemopreventive agent has been found to be insufficiently effective, and we have raised the possibility that a combination of agents would synergistically enhance the preventive effect of chemopreventive agents without enhancing their side effects. Random trials of combinations would be a waste time, and so we propose the need for a rational model, which we have developed and called the "combination-oriented molecular-targeting prevention" of cancer.

In earlier studies, we proposed the concept of "generegulating chemoprevention" as a model of the "molecular-targeting prevention" of cancer (Fig. 1a) [1-3]. In line with this concept, we have shown that chemoprevention can be realized by regulating the expression of a specific gene as a target molecule. Here, we propose a new concept - that a combination of different agents chosen on the basis of their molecular mechanisms is a useful approach in 
chemoprevention. We term this strategy "combinationoriented molecular-targeting prevention" of cancer. As shown in Fig. 1b, the administration of specific combinations of two agents can synergistically enhance their preventive effects, even if the effect of each separate agent of the combination is weak. Moreover, this approach may enhance only the preventive effects of the agents without increasing their side effects (Fig. 1c).

Tumor necrosis factor-related apoptosis-inducing ligand (TRAIL) is a promising anti-cancer agent [4-6]. However, as more than a few malignant tumor cell lines are resistant to TRAIL [7], it is necessary to overcome this resistance in order for chemotherapeutic programs using TRAIL to be reliable and effective. Many agents up-regulating the expression of death receptor (DR) 5, a TRAIL receptor, have been identified to date. These agents synergistically induce apoptosis in combination with TRAIL in malignant tumor cells; i.e., they overcome TRAIL resistance. In this review, we present our findings on the synergistic effects of TRAIL and various agents that up-regulate DR5 as a model of our novel concept, "combination-oriented molecular-targeting prevention" of cancer. We believe that this strategy can be applied to many other target molecules and that it will be extensively useful for cancer prevention.
A

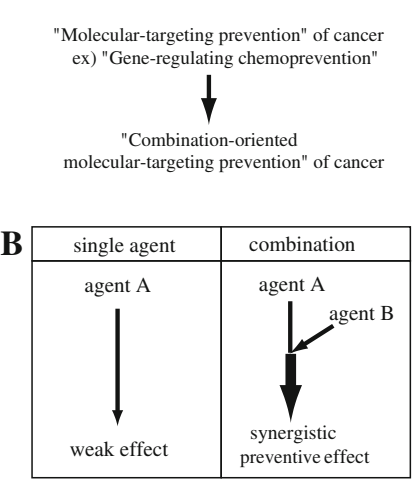

C
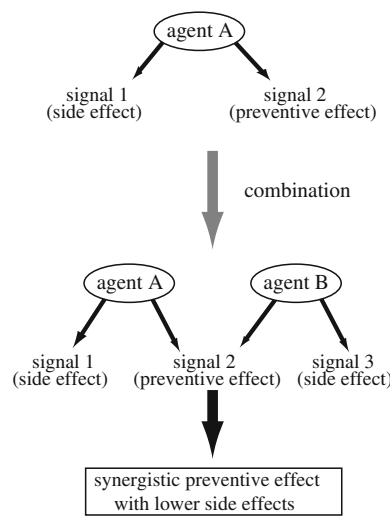

Fig. 1 The strategy of "combination-oriented molecular-targeting prevention" of cancer. a We previously proposed the concepts of "molecular-targeting prevention" of cancer and "gene-regulating chemoprevention". Based on these strategies, we propose here a new concept, namely, the "combination-oriented molecular-targeting prevention" of cancer, which is chemoprevention using a combination of agents that have been chosen based on their molecular mechanisms. b A single agent (agent $A$ ) exerts a weak effect. Agent A combined with another agent (agent $B$ ) generates synergistic preventive effects. c While the use of single agent A or B has a preventive effect and causes side effects, the combination of agent $\mathrm{A}$ and agent B synergistically enhances their preventive effect with relatively lower side effects

\section{Signaling pathway of TRAIL-mediated apoptosis}

TRAIL has been identified as an expressed sequenced tag (EST) that shows sequence homology to tumor necrosis factor (TNF) [4]. It is also known to be a cytokine belonging to the TNF superfamily. To date, five TRAIL specific receptors have been identified (Fig. 2). DR5 and DR4 are pro-apoptotic TRAIL receptors. DR5 is also called TRAILR2, TRICK2, or Killer [8-12], and DR4 is also called TRAIL-R1 [13]. As shown in Fig. 2, TRAIL interacts with cell surface DR5 or DR4 and forms a protein complex called the death-inducing signaling complex (DISC) by recruiting the adaptor protein FAS-associated death domain (FADD) and caspase- 8 or $-10[13,14]$. Caspase- 8 and -10 are auto-activated in DISC and cleave effector caspases, such as caspase-3. Effector caspases cleave many substrates including poly (ADP-ribose) polymerase (PARP) and, consequently, cause apoptosis. In certain cell types, caspase- 8 or -10 activity is not sufficient to activate effector caspases. Caspase- 8 and -10 cleave the bcl-2 interacting domain (Bid) [15], and truncated Bid causes the loss of mitochondrial membrane potential, cytochrome $\mathrm{C}$ release,

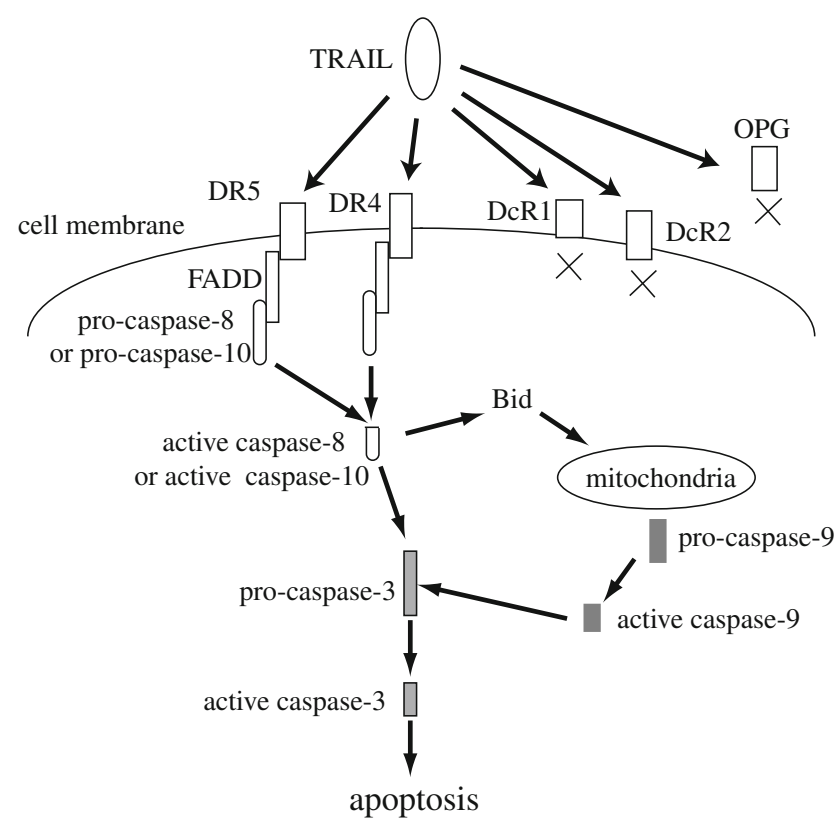

Fig. 2 Signaling pathway of tumor necrosis factor-related apoptosisinducing ligand (TRAIL). TRAIL interacts with death receptor 5 or 4 (DR5, DR4, respectively) on the cell surface and forms a protein complex termed death-inducing signaling complex (DISC) that contains the FAS-associated death domain protein $(F A D D)$ and procaspase- 8 or -10 . The activated caspase- 8 or -10 then cleaves and activates caspase- 3 and the bcl-2 interacting domain (Bid). Bid stimulates mitochondria and activates caspase- 9 , and the activated caspase-9 cleaves and activates caspase-3. Activated caspase-3 cleaves many substrates, resulting in apoptosis. Decoy receptor 1 and $2(D c R 1, D c R 2$, respectively) and osteoprotegerin $(O P G)$ have dominant negative functions and block TRAIL-induced apoptosis 
and caspase- 9 activation. Activated caspase- 9 cleaves and activates effector caspases, such as caspase-3, resulting in apoptosis. Other TRAIL receptors, such as decoy receptor (DcR) 1, DcR2 and osteoprotegerin (OPG), contain mutations or deletions in the intracellular death domain and are unable to transmit apoptotic signals $[13,14]$. These receptors act as dominant negative receptors and block the functions of DR5 and DR4 via interactions with TRAIL. Recombinant TRAIL induces apoptosis in cancer cells both in vitro and in vivo, but TRAIL has little or no toxicity in normal cells $[5,6]$. The possible mechanisms of the cancerspecific cytotoxicity of TRAIL are as follows. DcRs are expressed in normal cells at a higher level than in cancer cells, thereby preventing apoptosis caused by TRAIL through DR5 and DR4 pathways. The selective killing of cancer cells - but not normal cells - is an attractive property for cancer treatment, and for this reason TRAIL is considered to be a promising candidate as an anti-cancer agent. Recombinant TRAIL and agonistic antibodies against DR5 and DR4 are currently being tested in clinical studies as treatments against malignant tumors, but some types of malignant tumor cells remain tolerant to TRAIL [16-18]. Therefore, a new strategy for overcoming TRAIL resistance is required. On the other hand, TRAIL receptor deficiency promotes tumorigenesis and enhances metastasis in mice $[19,20]$. Therefore, pathways involving TRAIL and the above-mentioned receptors may be important for cancer prevention. We thus suggest that the up-regulation of DR5 or DR4 would be a useful strategy for cancer prevention and/or therapy.

\section{Combination of histone deacetylase inhibitors and TRAIL}

Histone deacetylase inhibitors (HDACIs) are novel anticancer agents; examples of HDACI are sodium butyrate, suberoylanilide hydroxamic acid (SAHA), trichostatin A (TSA), FK228, YM753 [21], valproic acid, among others [22]. HDACIs selectively alter gene transcription and induce cell cycle arrest and cell death in malignant tumor cells. They induce p21/WAF1 expression through a Sp1-binding site on its promoter independent of tumorsuppressor p53 and induce cell cycle arrest at the G1 phase in colon cancer cells [23-25]. Since sodium butyrate is a metabolite of dietary fibers, it is a model of gene-regulating chemoprevention [26]. HDACIs also up-regulate the INK4 family genes, p15, p18, and p19, through transcription, and the induction of these genes plays a role in G1 arrest [27-29]. HDACIs induce gadd45 through transcription mediated by both NF-Y and Oct-1 in a p53-independent manner, thereby causing G2/M arrest [30].
We found that HDACIs, such as sodium butyrate, TSA, and SAHA, induce the expression of the TRAIL receptor, DR5, in Jurkat T-cell leukemia cells (Fig. 3) [31]. Although treatment with either HDACI or TRAIL singly did not sufficiently induce apoptosis in Jurkat cells, the combined use of HDACIs together with TRAIL caused a drastic increase in apoptosis. As a possible mechanism, HDACIs may have up-regulated DR5 expression at the transcriptional level and consequently increased the sensitivity of the cells to TRAIL (Fig. 3). In conclusion, a combination treatment involving these two agents at suboptimal concentrations synergistically caused apoptosis by targeting the same molecular pathway. We therefore termed the strategy "combination-oriented molecular targeting prevention/therapy" of cancer.

\section{Combination of food components and TRAIL}

Food is one of the most important factors regulating the risk of carcinogenesis [32-34]. Epidemiological studies have elucidated that fruits and vegetables have the potential to prevent carcinogenesis in humans, and experimental studies have demonstrated that components in fruits and vegetables can prevent carcinogenesis in animals. Many recent reports have elucidated the molecular mechanisms by which various food components prevent carcinogenesis. We have also found that many chemopreventive food

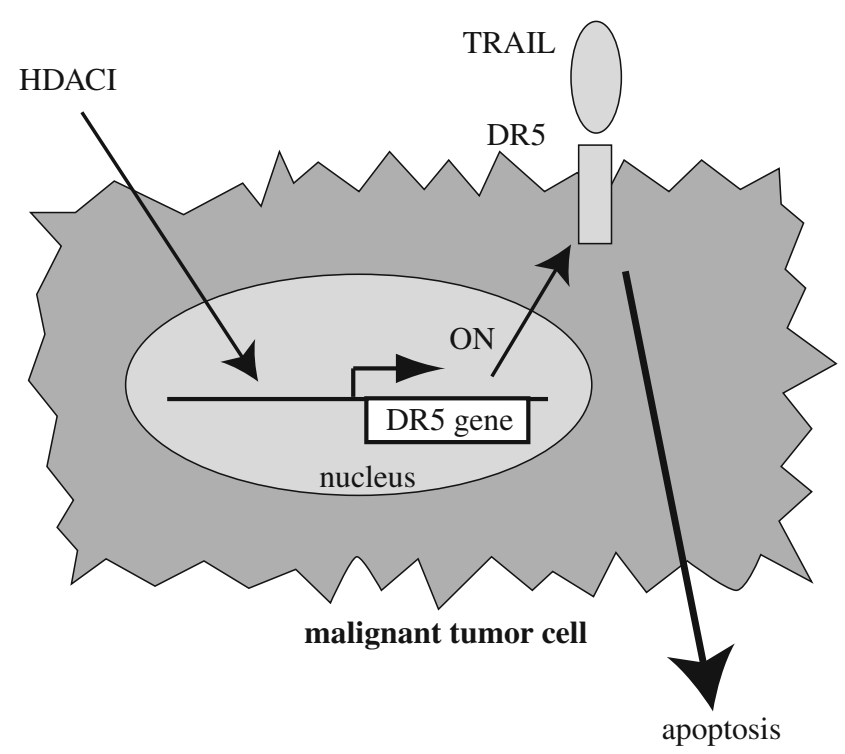

Fig. 3 Combination of histone deacetylase inhibitors (HDACIs) and TRAIL. HDACIs, such as sodium butyrate, trichostatin A (TSA), and suberoylanilide hydroxamic acid (SAHA) up-regulate DR5 expression through transcription, resulting in an increased level of the DR5 protein on the cell membrane and, consequently, an increased chance that TRAIL binds to DR5. The combination of HDACI and TRAIL can synergistically induce apoptosis in malignant tumor cells 
components induce the expression of molecules that inhibit carcinogenesis [1-3]. We therefore hypothesized that the combination of several components chosen on the basis of their molecular mechanisms would be useful for the prevention of cancer, which we termed "combination-oriented molecular-targeting prevention" of cancer. We found that many food components with cancer preventive effects upregulated DR5 expression and that when they were used together with TRAIL they synergistically and selectively induced apoptosis in malignant tumor cells (Fig. 4).

The natural flavone, luteolin, is found in edible plants, such as broccoli, celery, perilla leaves and seeds, and carrots, and has been reported to have preventive effects against cancer. In mice with fibrosarcoma induced by a carcinogen, luteolin-mixed diets were found to reduce tumor incidence and death [35]. In addition, topical application of luteolin prevented the promotion of skin papillomas in mice [36]. In in vitro experiments, luteolin has been reported to arrest the cell cycle at the G1 phase in human gastric cancer cells and to induce apoptosis in human leukemia cells [37, 38]. In our search for the mechanism behind the induction of apoptosis by luteolin, we found that luteolin up-regulated DR5 expression at the transcriptional level in human cervical carcinoma HeLa cells [39]. However, the apoptosis induced by luteolin alone was weak. We thus tried a combination of luteolin and TRAIL. As expected, luteolin combined with TRAIL synergistically induced apoptosis in HeLa cells [40]. Interestingly, the combination of luteolin and TRAIL did not induce apoptosis in normal human peripheral blood mononuclear cells (PBMCs). Similarly, another flavonoid, apigenin, which is contained in many fruits and vegetables,

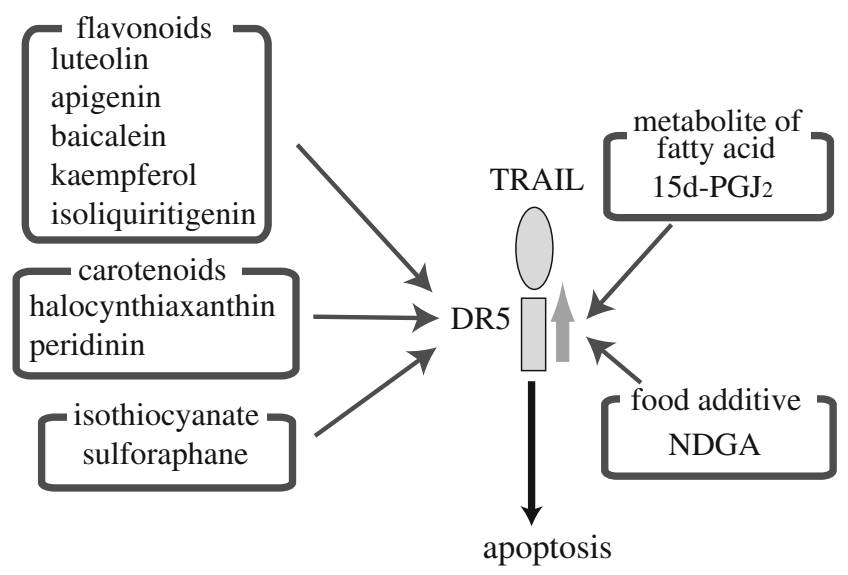

Fig. 4 Combination of food components and TRAIL. Flavonoids (luteolin, apigenin, baicalein, kaempferol, and isoliquiritigenin), carotenoids (halocynthiaxanthin and peridinin), isothiocyanate (sulforaphane), a metabolite of fatty acids, 15 -deoxy- $\Delta^{12,14}$-prostaglandin $\mathrm{J}_{2}\left(15 d-P G J_{2}\right)$, and a food additive $(N D G A)$ up-regulate DR5 expression. Combining these food components with TRAIL drastically induces apoptosis in malignant tumor cells had synergistic effects when used together with TRAIL in Jurkat T-cell leukemia cells but not in normal human PBMCs [41]. Apigenin also up-regulated DR5 expression at the protein level but not DR5 promoter or mRNA levels in Jurkat cells. We also found that apigenin increased DR5 protein stability in Jurkat cells.

Not only flavonoids but also carotenoids are able to induce apoptosis in malignant tumor cells when used together with TRAIL. Carotenoids are naturally occurring compounds contained in plants and microorganisms [34, 42], and animals require carotenoids in their diet since they can not synthesize them. Results from epidemiological and animal studies have indicated that a variety of carotenoids have anti-carcinogenic effects. Halocynthiaxanthin is a carotenoid contained in sea squirts and oysters that causes apoptosis [43], inhibits tumor promotion by Epstein-Barr virus (EBV) [44], and suppresses free radical generation [45]. Peridinin is also a carotenoid extracted from corbicula clams. Halocynthiaxanthin and peridinin increase DR5 mRNA and protein levels, and a combination of TRAIL and either of the carotenoids was found to strongly induce apoptosis in human colon cancer cells [46].

Isothiocyanate sulforaphane is contained in cruciferous vegetables, such as broccoli [47]. Sulforaphane is known to prevent chemically induced carcinogenesis in colon and stomach tumors [48, 49], and it also shows chemotherapeutic potential in prostate and breast cancer xenografts of mice [50, 51]. We found that sulforaphane up-regulated DR5 expression and enhanced TRAIL-induced apoptosis in human osteosarcoma cells [52].

Arachidonic acid is a polyunsaturated fatty acid and one of the essential fatty acids. In addition, arachidonic acid is released from membrane phospholipids by the action of phospholipases and synthesized into prostaglandins, which are a family of naturally occurring cyclic 20-carbon fatty acids [53]. One of its terminal metabolites, 15-deoxy$\Delta^{12,14}$-prostaglandin $\mathrm{J}_{2}\left(15 \mathrm{~d}-\mathrm{PGJ}_{2}\right)$, inhibits the proliferation of malignant tumor cells due to its ability to arrest G1 cell cycle through the mediation of $\mathrm{p} 15^{\mathrm{INK} 4 \mathrm{~b}}$ up-regulation [54]. Moreover, we found that $15 \mathrm{~d}_{-} \mathrm{PGJ}_{2}$ up-regulated DR5 expression and that a combination of $15 \mathrm{~d}-\mathrm{PGJ}_{2}$ and TRAIL synergistically induced apoptosis in Jurkat T-cell leukemia cells and prostate cancer PC3 cells [55].

Nordihydroguaiaretic acid (NDGA) is a food additive obtained from plants that inhibits lipoxygenases, which induce tumor cell proliferation, metastasis, and angiogenesis [56, 57]. NDGA is also able to prevent mitogeninduced carcinogenesis and attenuate the growth of human cancer xenografts [58, 59]. We have shown that NDGA increases DR5 mRNA and protein levels, thereby enhancing TRAIL sensitivity of human T-cell leukemia Jurkat cells, prostate cancer DU145 cells, and colon cancer SW480 cells [60]. Since another lipoxygenase inhibitor, 
AA861, also shows the same effects, we strongly suggest that inhibition of the lipoxygenase pathway causes the upregulation of DR5 expression [60].

\section{Combination of CCAAT/enhancer binding protein homologous protein inducers and TRAIL}

When developing strategies such as the "combinationoriented molecular-targeting prevention" of cancer, it is essential to have detailed knowledge of the molecular mechanisms of the combined agents. We therefore examined the mechanisms by which DR5 inducers up-regulate DR5 expression and found that several agents were able to up-regulate DR5 through the transcription factor CCAAT/ enhancer binding protein homologous protein (CHOP) (Fig. 5). We further determined that these agents were able to synergistically enhance the apoptosis evoked by TRAIL (Fig. 5).

Fenretinide [ $N$-(4-hydroxyphenyl]retinamide, 4HPR] is a semisynthetic retinoid that acts in both a retinoic acid receptor (RAR)-dependent and -independent manner [61]. Fenretinide is considered to have a greater therapeutic ability than classical retinoic acids due to its potential to induce apoptosis rather than differentiation and to have synergistic effects with conventional chemotherapeutic agents, such as cisplatin or etoposide [62]. In addition, fenretinide has been tested in clinical trials including phase III trials as a cancer chemopreventive agent [63]. We examined the effects of fenretinide on DR genes and found that fenretinide up-regulated DR5 expression by enhancing its promoter activity [64]. Subsequent promoter analysis revealed that the transcription factor $\mathrm{CHOP}$ was

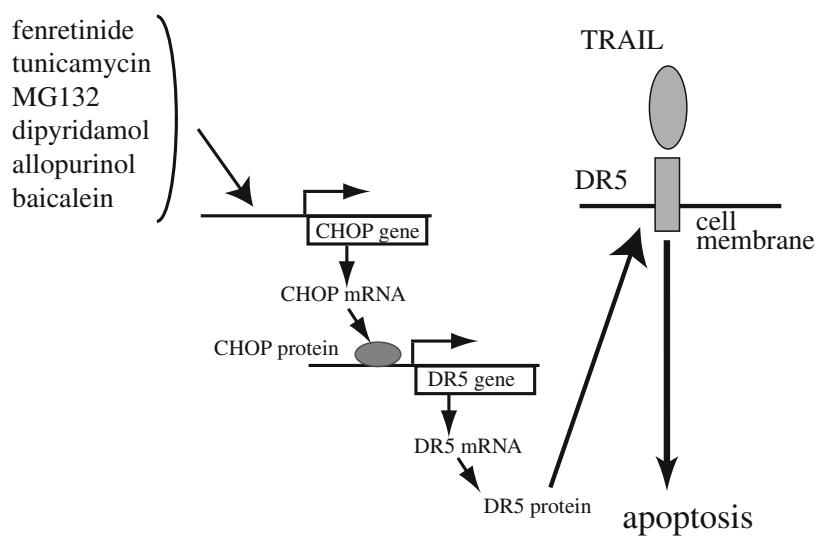

Fig. 5 Combination of CCAAT/enhancer binding protein homologous protein $(\mathrm{CHOP})$ inducers and TRAIL. CHOP is a transcription factor that up-regulates DR5 expression, and CHOP inducers, such as fenretinide, tunicamycin, MG132, dipyridamol, allopurinol, and baicalein, also up-regulate DR5 expression by inducing CHOP. Therapeutic treatment combining these agents and TRAIL can synergistically induce apoptosis in malignant tumor cells responsible for the up-regulation of DR5 by fenretinide. Fenretinide also enhanced TRAIL sensitivity in human colon cancer cells via the up-regulation of DR5. The $\mathrm{N}$-linked glycosylation inhibitor tunicamycin, the proteasome inhibitor MG132, the anti-platelet agent dipyridamole, the anti-gout agent allopurinol, and baicalein, a flavonoid derived from the roots of Scutellaria baicalensis used in Chinese herbal medicine, have also been reported to up-regulate DR5 by enhancing CHOP expression, thereby sensitizing cancer cells to TRAIL-induced apoptosis [65-69]. As described above, CHOP is an important molecular target in the up-regulation of DR5 as a way of enhancing TRAIL efficacy. Thus, if the underlying molecular mechanisms of combined agents are revealed, other combinations of agents will be easily found based on their mechanisms.

\section{Future prospects}

Here, we have demonstrated that many agents, such as HDACI, food components, and CHOP inducers, are able to increase the expression of the TRAIL receptor DR5 and facilitate TRAIL sensitivity in malignant tumor cells. Combinations of TRAIL and these agents are able to synergistically induce apoptosis at sub-optimal concentrations of each agent. It is very encouraging that such combinations can even kill TRAIL-resistant cancer cells. The combination strategy involving the TRAIL-DR5 pathway is therefore promising for "combination-oriented molecular-targeting prevention" of cancer.

In this review, we have shown that membrane receptors could be molecular targets for the strategy "combinationoriented molecular-targeting prevention" of cancer. As shown in Fig. 6a, we also raise the possibility that molecules other than membrane receptors can be used for the "combination-oriented molecular-targeting prevention" of cancer. Using this scheme, we found that not only agents that up-regulate the expression of the receptor (agent $\mathrm{A}$ in Fig. 6a) but also those agents that activate the downstream pathway of the receptor (agent B in Fig. 6a) are useful for the prevention of cancer.

As a chemopreventive agent, TRAIL itself is not suitable because it is a protein product. Therefore, agents that up-regulate endogenous TRAIL would be very useful in cancer prevention strategies. On the other hand, TRAIL is an endogeneous cytokine and functions in immune responses [13, 14]. It has recently been reported that interferon $\alpha$, interferon $\gamma$, retinoic acid, or Bacillus Calmette-Guerin can increase endogeneous TRAIL expression and release soluble TRAIL from cancer or immune cells [70-72]. We therefore expect that foods, beverages, and supplements containing both a TRAIL inducer and a DR5 

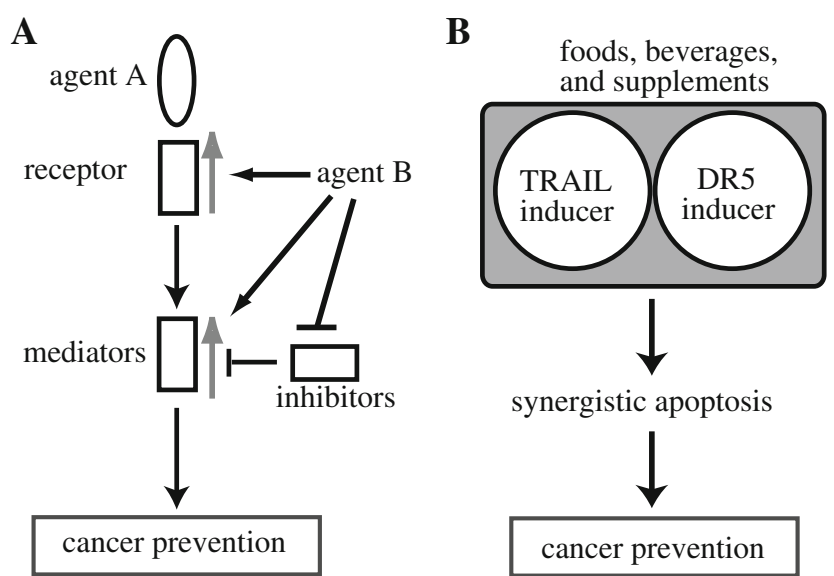

Fig. 6 Future prospects of the strategy "combination-oriented molecular-targeting prevention" of cancer. a Various molecules other than membrane receptors could act as molecular targets for the strategy "combination-oriented molecular-targeting prevention" of cancer. b Foods, beverages, and supplements containing both a TRAIL inducer and a DR5 inducer may prevent carcinogenesis

inducer would be able to synergistically induce apoptosis, resulting in the prevention of carcinogenesis and the "combination-oriented molecular-targeting prevention" of cancer (Fig. 6b).

We have presented a new concept for a chemoprevention strategy that involves a combination of agents being used to target specific molecules. We have also proposed that the combination of DR5 inducers and TRAIL is promising as the model. We further suggest that the strategy "combination-oriented molecular-targeting prevention" of cancer could be used as a prototype for cancer prevention in the future and that it would be a useful approach for targeting other signaling pathways as a way of treating other diseases.

Acknowledgments This work was supported in part by the Japanese Ministry of Education, Culture, Sports, Science, and Technology. We also thank all contributors.

\section{References}

1. Sowa Y, Sakai T. Gene-regulating chemoprevention against cancer-as a model for "molecular-targeting prevention" of cancer (in Japanese). Nippon Eiseigaku Zasshi. 2003;58:267-74.

2. Matsuzaki Y, Sowa Y, Hirose T, Yokota T, Sakai T. Histone deacetylase inhibitors-promising agents for "gene-regulating chemoprevention" and "molecular-targeting prevention" of cancer. Environ Health Prev Med. 2003;8:157-60.

3. Matsuzaki Y, Sakai T. INK4 family-a promising target for "gene-regulating chemoprevention" and "molecular-targeting prevention" of cancer. Environ Health Prev Med. 2005;10:72-7.

4. Pitti RM, Marsters SA, Ruppert S, Donahue CJ, Moore A, Ashkenazi A. Induction of apoptosis by Apo-2 ligand, a new member of the tumor necrosis factor cytokine family. J Biol Chem. 1996;271:12687-90.
5. Walczak H, Miller RE, Ariail K, Gliniak B, Griffith TS, Kubin M, et al. Tumoricidal activity of tumor necrosis factor-related apoptosis-inducing ligand in vivo. Nat Med. 1999;5:157-63.

6. Ashkenazi A, Pai RC, Fong S, Leung S, Lawrence DA, Marsters SA, et al. Safety and antitumor activity of recombinant soluble Apo2 ligand. J Clin Invest. 1999;104:155-62.

7. Zhang L, Fang B. Mechanisms of resistance to TRAIL-induced apoptosis in cancer. Cancer Gene Ther. 2005;12:228-37.

8. Pan G, Ni J, Wei Y-F, Yu G-L, Gentz R, Dixit VM. An antagonist decoy receptor and a death domain-containing receptor for TRAIL. Science. 1997;277:815-8.

9. Sheridan JP, Marsters SA, Pitti RM, Gurney A, Skubatch M, Baldwin D, et al. Control of TRAIL-induced apoptosis by a family of signaling and decoy receptors. Science. 1997;277:818 21.

10. Screaton GR, Mongkolsapaya J, Xu X-N, Cowper AE, McMichael AJ, Bell JI. TRICK2, a new alternatively spliced receptor that transduces the cytotoxic signal from TRAIL. Curr Biol. 1997;7:693-6.

11. Walczak H, Degli-Esposti MA, Johnson RS, Smolak PJ, Waugh JY, Boiani N, et al. TRAIL-R2: a novel apoptosis-mediating receptor for TRAIL. EMBO J. 1997;16:5386-97.

12. Wu GS, Burns TF, McDonald ER 3rd, Jiang W, Meng R, Krantz ID, et al. KILLER/DR5 is a DNA damage-inducible p53-regulated death receptor gene. Nat Genet. 1997;17:141-3.

13. LeBlanc HN, Ashkenazi A. Apo2L/TRAIL and its death and decoy receptors. Cell Death Differ. 2003;10:66-75.

14. Takeda K, Stagg J, Yagita H, Okumura K, Smyth MJ. Targeting death-inducing receptors in cancer therapy. Oncogene. 2007; 26:3745-57.

15. Yamada H, Tada-Oikawa S, Uchida A, Kawanishi S. TRAIL causes cleavage of bid by caspase- 8 and loss of mitochondrial membrane potential resulting in apoptosis in BJAB cells. Biochem Biophys Res Commun. 1999;265:130-3.

16. Ichikawa K, Liu W, Zhao L, Wang Z, Liu D, Ohtsuka T, et al. Tumoricidal activity of a novel anti-human DR5 monoclonal antibody without hepatocyte cytotoxicity. Nat Med. 2001;7:95460.

17. Pukac L, Kanakaraj P, Humphreys R, Alderson R, Bloom M, Sung C, et al. HGS-ETR1, a fully human TRAIL-receptor 1 monoclonal antibody, induces cell death in multiple tumour types in vitro and in vivo. Br J Cancer. 2005;92:1430-41.

18. Fesik SW. Promoting apoptosis as a strategy for cancer drug discovery. Nat Rev Cancer. 2005;5:876-85.

19. Grosse-Wilde A, Voloshanenko O, Bailey SL, Longton GM, Schaefer U, Csernok AI, et al. TRAIL-R deficiency in mice enhances lymph node metastasis without affecting primary tumor development. J Clin Invest. 2008;118:100-10.

20. Finnberg N, Klein-Szanto AJ, El-Deiry WS. TRAIL-R deficiency in mice promotes susceptibility to chronic inflammation and tumorigenesis. J Clin Invest. 2008;118:111-23.

21. Shindoh N, Mori M, Terada Y, Oda K, Amino N, Kita A, et al. YM753, a novel histone deacetylase inhibitor, exhibits antitumor activity with selective, sustained accumulation of acetylated histones in tumors in the WiDr xenograft model. Int $\mathrm{J}$ Oncol. 2008;32:545-55

22. Xu WS, Parmigiani RB, Marks PA. Histone deacetylase inhibitors: molecular mechanisms of action. Oncogene. 2007;26:554152.

23. Sowa Y, Orita T, Minamikawa S, Nakano K, Mizuno T, Nomura $\mathrm{H}$, et al. Histone deacetylase inhibitor activates the WAF1/Cip1 gene promoter through the Sp1 sites. Biochem Biophys Res Commun. 1997;241:142-50.

24. Nakano K, Mizuno T, Sowa Y, Orita T, Yoshino T, Okuyama Y, et al. Butyrate activates the WAF1/Cip1 gene promoter through 
Sp1 sites in a p53-negative human colon cancer cell line. J Biol Chem. 1997;272:22199-206.

25. Sowa Y, Orita T, Minamikawa-Hiranabe S, Mizuno T, Nomura H, Sakai T. Sp3, but not Sp1, mediates the transcriptional activation of the $\mathrm{p} 21 / \mathrm{WAF} 1 / \mathrm{Cip} 1$ gene promoter by histone deacetylase inhibitor. Cancer Res. 1999;59:4266-70.

26. Sowa Y, Sakai T. Butyrate as a model for "gene-regulating chemoprevention and chemotherapy". Biofactors. 2000;12:283-7.

27. Hitomi T, Matsuzaki Y, Yokota T, Takaoka Y, Sakai T. p15 $5^{\text {INK4b }}$ in HDAC inhibitor-induced growth arrest. FEBS Lett. 2003; 554:347-50.

28. Yokota T, Matsuzaki Y, Sakai T. Trichostatin A activates p1 $8^{\text {INK4c }}$ gene: differential activation and cooperation with p19 ${ }^{\text {INK4d }}$ gene. FEBS Lett. 2004;574:171-5.

29. Yokota T, Matsuzaki Y, Miyazawa K, Zindy F, Roussel MF, Sakai T. Histone deacetylase inhibitors activate INK4d gene through Sp1 site in its promoter. Oncogene. 2004;23:5340-9.

30. Hirose T, Sowa Y, Takahashi S, Saito S, Yasuda C, Shindo N, et al. p53-Independent induction of Gadd45 by histone deacetylase inhibitor: coordinate regulation by transcription factors Oct-1 and NF-Y. Oncogene. 2003;22:7762-73.

31. Nakata S, Yoshida T, Horinaka M, Shiraishi T, Wakada M, Sakai T. Histone deacetylase inhibitors upregulate death receptor 5/ TRAIL-R2 and sensitize apoptosis induced by TRAIL/APO2-L in human malignant tumor cells. Oncogene. 2004;23:6261-71.

32. Shukla S, Gupta S. Dietary agents in the chemoprevention of prostate cancer. Nutr Cancer. 2005;53:18-32.

33. Taghavi N, Yazdi I. Type of food and risk of oral cancer. Arch Iran Med. 2007;10:227-32.

34. Nishino H, Murakoshi M, Ii T, Takemura M, Kuchide M, Kanazawa M, et al. Carotenoids in cancer chemoprevention. Cancer Metastasis Rev. 2002;21:257-64.

35. Elangovan V, Sekar N, Govindasamy S. Chemopreventive potential of dietary bioflavonoids against 20-methylcholanthreneinduced tumorigenesis. Cancer Lett. 1994;87:107-13.

36. Ueda H, Yamazaki C, Yamazaki M. Inhibitory effect of Perilla leaf extract and luteolin on mouse skin tumor promotion. Biol Pharm Bull. 2003;26:560-3.

37. Matsukawa Y, Marui N, Sakai T, Satomi Y, Yoshida M, Matsumoto K, et al. Genistein arrests cell cycle progression at G2-M. Cancer Res. 1993;53:1328-31.

38. Ko WG, Kang TH, Lee SJ, Kim YC, Lee BH. Effects of luteolin on the inhibition of proliferation and induction of apoptosis in human myeloid leukaemia cells. Phytother Res. 2002;16:295-8.

39. Horinaka M, Yoshida T, Shiraishi T, Nakata S, Wakada M, Nakanishi R, et al. Luteolin induces apoptosis via death receptor 5 upregulation in human malignant tumor cells. Oncogene. 2005;24:7180-9.

40. Horinaka M, Yoshida T, Shiraishi T, Nakata S, Wakada M, Nakanishi R, et al. The combination of TRAIL and luteolin enhances apoptosis in human cervical cancer HeLa cells. Biochem Biophys Res Commun. 2005;333:833-8.

41. Horinaka M, Yoshida T, Shiraishi T, Nakata S, Wakada M, Sakai $\mathrm{T}$. The dietary flavonoid apigenin sensitizes malignant tumor cells to tumor necrosis factor-related apoptosis-inducing ligand. Mol Cancer Ther. 2006;5:945-51.

42. Krinsky NI, Johnson EJ. Carotenoid actions and their relation to health and disease. Mol Aspects Med. 2005;26:459-516.

43. Nishino H, Tsushima M, Matsuno T, Tanaka Y, Okuzumi J, Murakoshi M, et al. Anti-neoplastic effect of halocynthiaxanthin, a metabolite of fucoxanthin. Anticancer Drugs. 1992;3:493-7.

44. Tsushima M, Maoka T, Katsuyama M, Kozuka M, Matsuno T, Tokuda $\mathrm{H}$, et al. Inhibitory effect of natural carotenoids on Epstein-Barr virus activation activity of a tumor promoter in Raji cells. A screening study for anti-tumor promoters. Biol Pharm Bull. 1995;18:227-33.
45. Murakami A, Nakashima M, Koshiba T, Maoka T, Nishino H, Yano M, et al. Modifying effects of carotenoids on superoxide and nitric oxide generation from stimulated leukocytes. Cancer Lett. 2000;149:115-23.

46. Yoshida T, Maoka T, Das SK, Kanazawa K, Horinaka M, Wakada $M$, et al. Halocynthiaxanthin and peridinin sensitize colon cancer cell lines to tumor necrosis factor-related apoptosisinducing ligand. Mol Cancer Res. 2007;5:615-25.

47. Fahey JW, Zhang Y, Talalay P. Broccoli sprouts: an exceptionally rich source of inducers of enzymes that protect against chemical carcinogens. Proc Natl Acad Sci USA. 1997;94:1036772.

48. Chung FL, Conaway CC, Rao CV, Reddy BS. Chemoprevention of colonic aberrant crypt foci in Fischer rats by sulforaphane and phenethyl isothiocyanate. Carcinogenesis. 2000;21:2287-91.

49. Fahey JW, Haristoy X, Dolan PM, Kensler TW, Scholtus I, Stephenson KK, et al. Sulforaphane inhibits extracellular, intracellular, and antibiotic-resistant strains of Helicobacter pylori and prevents benzo[a]pyrene-induced stomach tumors. Proc Natl Acad Sci USA. 2002;99:7610-5.

50. Singh AV, Xiao D, Lew KL, Dhir R, Singh SV. Sulforaphane induces caspase-mediated apoptosis in cultured PC-3 human prostate cancer cells and retards growth of PC-3 xenografts in vivo. Carcinogenesis. 2004;25:83-90.

51. Jackson SJ, Singletary KW. Sulforaphane: a naturally occurring mammary carcinoma mitotic inhibitor, which disrupts tubulin polymerization. Carcinogenesis. 2004;25:219-27.

52. Matsui TA, Sowa Y, Yoshida T, Murata H, Horinaka M, Wakada $M$, et al. Sulforaphane enhances TRAIL-induced apoptosis through the induction of DR5 expression in human osteosarcoma cells. Carcinogenesis. 2006;27:1768-77.

53. Straus DS, Glass CK. Cyclopentenone prostaglandins: new insights on biological activities and cellular targets. Med Res Rev. 2001;21:185-210.

54. Matsuzaki Y, Koyama M, Hitomi T, Takaoka Y, Kawanaka M, Sakai T. 15-Deoxy- $\Delta^{12,14}$-prostaglandin J2 activates the expression of p15INK4b gene, a cyclin-dependent kinase inhibitor. Int J Oncol. 2005;27:497-503.

55. Nakata S, Yoshida T, Shiraishi T, Horinaka M, Kouhara J, Wakada M, et al. 15-Deoxy- $\Delta^{12,14}$-prostaglandin J2 induces death receptor 5 expression through mRNA stabilization independently of PPAR $\gamma$ and potentiates TRAIL-induced apoptosis. Mol Cancer Ther. 2006;5:1827-35.

56. Hagmann W. Lipoxygenase in human tumor cells. Pathol Oncol Res. 1997;3:83-8.

57. Shureiqi I, Lippman SM. Lipoxygenase modulation to reverse carcinogenesis. Cancer Res. 2001;61:6307-12.

58. Yu A, Hashimura T, Nishio Y, Kanamaru H, Fukuzawa S, Yoshida O. Anti-promoting effect of nordihydroguaiaretic acid on $N$-butyl- $N$-(4-hydroxybutyl)nitrosamine and sodium saccharin-induced rat urinary bladder carcinogenesis. Jpn J Cancer Res. 1992;83:944-8.

59. Tong WG, Ding XZ, Witt RC, Adrian TE. Lipoxygenase inhibitors attenuate growth of human pancreatic cancer xenografts and induce apoptosis through the mitochondrial pathway. Mol Cancer Ther. 2002;1:929-35.

60. Yoshida T, Shiraishi T, Horinaka M, Nakata S, Yasuda T, Goda $\mathrm{AE}$, et al. Lipoxygenase inhibitors induce death receptor 5/ TRAIL-R2 expression and sensitize malignant tumor cells to TRAIL-induced apoptosis. Cancer Sci. 2007;98:1417-23.

61. Clifford JL, Menter DG, Wang M, Lotan R, Lippman SM. Retinoid receptor-dependent and -independent effects of $\mathrm{N}$-(4hydroxyphenyl)retinamide in F9 embryonal carcinoma cells. Cancer Res. 1999;59:14-8.

62. Lovat PE, Ranalli M, Bernassola F, Tilby M, Malcolm AJ, Pearson $\mathrm{AD}$, et al. Synergistic induction of apoptosis of 
neuroblastoma by fenretinide or CD437 in combination with chemotherapeutic drugs. Int J Cancer. 2000;88:977-85.

63. Malone W, Perloff M, Crowell J, Sigman C, Higley H. Fenretinide: a prototype cancer prevention drug. Expert Opin Investig Drugs. 2003;12:1829-42.

64. Kouhara J, Yoshida T, Nakata S, Horinaka M, Wakada M, Ueda $\mathrm{Y}$, et al. Fenretinide up-regulates DR5/TRAIL-R2 expression via the induction of the transcription factor $\mathrm{CHOP}$ and combined treatment with fenretinide and TRAIL induces synergistic apoptosis in colon cancer cell lines. Int J Oncol. 2007;30:679-87.

65. Shiraishi T, Yoshida T, Nakata S, Horinaka M, Wakada M, Mizutani $\mathrm{Y}$, et al. Tunicamycin enhances tumor necrosis factorrelated apoptosis-inducing ligand-induced apoptosis in human prostate cancer cells. Cancer Res. 2005;65:6364-70.

66. Yoshida T, Shiraishi T, Nakata S, Horinaka M, Wakada M, Mizutani Y, et al. Proteasome inhibitor MG132 induces death receptor 5 through CCAAT/enhancer-binding protein homologous protein. Cancer Res. 2005;65:5662-7.

67. Goda AE, Yoshida T, Horinaka M, Yasuda T, Shiraishi T, Wakada M, et al. Mechanisms of enhancement of TRAIL tumoricidal activity against human cancer cells of different origin by dipyridamole. Oncogene. 2008;27:3435-45.
68. Yasuda T, Yoshida T, Goda AE, Horinaka M, Yano K, Shiraishi $\mathrm{T}$, et al. Anti-gout agent allopurinol exerts cytotoxicity to human hormone-refractory prostate cancer cells in combination with TRAIL. Mol Cancer Res. 2008;6:1852-60.

69. Taniguchi T, Yoshida T, Horinaka M, Yasuda T, Goda AE, Konishi M, et al. Baicalein overcomes TRAIL resistance via two different cell specific pathways in cancer cells but not in normal cells. Cancer Res. 2008;68:8918-27.

70. Tecchio C, Huber V, Scapini P, Calzetti F, Margotto D, Todeschini $\mathrm{G}$, et al. IFN $\alpha$-stimulated neutrophils and monocytes release a soluble form of TNF-related apoptosis-inducing ligand (TRAIL/Apo-2 ligand) displaying apoptotic activity on leukemic cells. Blood. 2004;103:3837-44.

71. Clarke N, Jimenez-Lara AM, Voltz E, Gronemeyer H. Tumor suppressor IRF-1 mediates retinoid and interferon anticancer signaling to death ligand TRAIL. EMBO J. 2004;23:3051-60.

72. Ludwig AT, Moore JM, Luo Y, Chen X, Saltsgaver NA, O'Donnell MA, et al. Tumor necrosis factor-related apoptosisinducing ligand: a novel mechanism for Bacillus CalmetteGuerin-induced antitumor activity. Cancer Res. 2004;64:3386-90. 